\title{
Design and fabrication of high performance photovoltaic based Controllable SnS/rGO hybrid composite: An excellent counter electrode for dye sensitized solar cell
}

M. Indhumathy

Thiruvalluvar Government Arts College

A Praksam ( $\sim$ aprakasam2020@gmail.com )

Thiruvalluvar Arts College https://orcid.org/0000-0003-4820-5091

H. H. Hegazy

Al-Azhar University

\section{Research Article}

Keywords: SnS, Reduced graphene oxide, Counter electrode, DSSC, Solar cell

Posted Date: March 30th, 2021

DOl: https://doi.org/10.21203/rs.3.rs-329169/v1

License: (c) (i) This work is licensed under a Creative Commons Attribution 4.0 International License.

Read Full License 


\section{Abstract}

To catalyse the decrease of triiodide in the electrolyte, state-of-the-art dye-sensitized sun directed cells (DSSCs) usually use respectable and uncommon Pt as counter electrodes, which greatly limits the practical applications of DSSCs. Appropriately, choices with low cost, fabulous electro- catalytic action, and predominant electrochemical stability to Pt are exceedingly looked for after. Herein, we report tin sulfide/reduced graphene oxide ( $\mathrm{SnS} / \mathrm{rGO}$ ) composites are facile synthesized via a simple one-pot hydrothermal route and characterized by XRD, FESEM, Raman, UV-DRS, BET and PL studies. Due to the synergic effect between the SnS nanoparticles and $\mathrm{rGO}$, these composite samples showed outstanding electro-catalytic property on reducing the triiodide. Therefore, the DSSC equipped with SnS/rGO nanocomposite CE achieved a power conversion efficiency (PCE) of $7.72 \%$, which was higher than those of use SnS nanoparticles (5.21\%). The improved mechanism of SnS/rGO nanocomposite was also discussed.

\section{Introduction}

At the turn of the century, the energy condition has been in a state of flux. People are searching for alternative sources of electricity. Solar energy has a lot of potential as a possible source of energy [1]. Because of their high performance, easy fabrication process, pollution-free design, and low consumption, DSSCs have sparked a lot of interest and large-scale research in a lot of academic labs [2]. The DSSC had a translucent conducting optical glass, photoanode, dyes, electrolyte, and counter electrode (CE) as a standard structure [3, 4]. Counter electrodes (CEs) are important in DSSCs because they capture electrons from the conduction band of photoanodes and catalyse the reduction of triiodide $\left(\mathrm{I}_{3}{ }^{-}\right)$to iodide $(\mathrm{I}-\mathrm{)}$ [5]. In any case, the use of respectable metal Pt as CEs, which is costly, resource-limited, and electrochemically instable, limits the large-scale applications of DSSCs [6,7]. Carbon materials are widely considered to be attractive candidates for Pt CEs because of their inherently low cost, easy usability, wide surface area, and high solidity in an electrochemical environment [8-10]. Graphene, a one-atom-thick two-dimensional sheet of sp2- bonded crystal structure with a range of great properties such as tall carrier mobility, electric conductivity, thermal conductivity, and transparency, may be a new member of the carbon crystal family among the numerous carbon-based materials [11]. It was also used in the construction of the DSSC as CE [12]. Despite the fact that bare graphene has a poor photovoltaic conversion efficiency due to the two distinct regions that influence bulk electrochemical operation, the basal planes and the edge planes, bare graphene has a high photovoltaic conversion efficiency. Combining hybrid composites is an efficient way to increase photo conversion performance and overcome this drawback. Transitional metal dichalcogenides (TMDs) have sparked a lot of interest because of their peculiar layered structure and complex electronic and optical properties [13-15]. Tin sulphide (SnS) is one of the most common metal chalcogenides $\left(\mathrm{MoS}_{2}, \mathrm{TiS}, \mathrm{ZnS}, \mathrm{WS}_{2}\right)$ among the various forms of metal chalcogenides $\left(\mathrm{MoS}_{2}, \mathrm{TiS}, \mathrm{ZnS}\right.$, $W_{2}$ ). Chemical stability, superior mechanical and optical properties, and stable electrical conductivity are all advantages. Moreover, combination of $\mathrm{SnS} / \mathrm{rGO}$ can retain the favorable properties of graphene (stated above) and expand additional favorable properties such as better electrical conductivity for faster 
charge transfer and induced defect sites for photovoltaic devices. Hence, we report SnS/rGO nanocomposite by a facile hydrothermal route and fabricate sandwich type DSSC based device. To the best of the authors knowledge, this is the first report about high performance dye DSSC based CE using $\mathrm{SnS} / \mathrm{rGO}$ composite via a facile hydrothermal route.

\section{Materials And Methods}

\subsection{Materials}

Tin(II) acetate (purity $>99.0 \%$ ), thioacetamide (purity $>99.0 \%$ ), and natural graphite flakes (size $<45 \mathrm{~mm}$, purity 99.99\%) were purchased from Sigma Aldrich (USA). Fluorine doped tin oxide (FTO) conducting glass substrates were bought from Hind High Vacuum (HHV). The glass wares were washed systematically and dried in hot air oven previous to use.

\subsection{Preparation of rGO and SnS/rGO nanocomposite}

GO was synthesized by the modified Hummers' method [16]. In a typical synthesis of bare SnS, $0.5 \mathrm{~g}$ of tin(II) acetate was dissolved in $50 \mathrm{~mL}$ of ethylene glycol (EG). Then $50 \mathrm{ml}$ of

Thioacetamide was added above solution and stirred for $1 \mathrm{~h}$ until the homogenous solution was obtained. The resultant solution transferred into a Teflon-lined stainless steel autoclave and then heated in an electric oven at $180^{\circ} \mathrm{C}$ for $24 \mathrm{~h}$. Finally, the autoclave was allowed to cool down to room temperature and the precipitate was collected by centrifugation, washed several times with absolute ethanol, and dried at $60^{\circ} \mathrm{C}$ in vacuum overnight. In the synthesis process of SnS/rGO, different weight percentage of (5, 10 and 15\%) GO was dissolved in the tin precursor and the experiment was done for identical condition. The prepared pure SnS and SnS/rGO (5, 10 and 15\%) samples were labeled by SnS, SRG-5, SRG-10 and SRG-15, respectively.

\subsection{DSSC fabrication setup}

The present solar cell device is sandwich type, which is consists of photoanode, cathode and electrolyte solution, respectively. The photoanode and cathode of DSSC is fabricated using SnS/rGO and commercial platinum (Pt) paste deposition on FTO substrate using doctor blade method. After preparation of photoanode was immersed in N719 dye solution. $0.6 \mathrm{M}$ iodide/triiodide liquid electrolyte injected between photoanode and cathode, respectively. AM1.5, $100 \mathrm{mWcm}^{-2}$ solar stimulator was used to monitor the photocurrent vs. voltage characteristics. The active surface area of the DSSC was 0.16 $\mathrm{cm}^{2}\left(0.4 \times 0.4 \mathrm{~cm}^{2}\right)$.

\section{Results And Discussion}

\subsection{XRD analysis}


The powder X-ray diffraction patterns of pure GO, SnS, and SnS/rGO nanohybrid samples were shown in Figure 1 respectively. The peak is situated at $10.89^{\circ}$, which corresponds to the (002) plane of GO and has an interplanar spacing of $0.85 \mathrm{~nm}$, as seen in Fig.1. All diffraction peaks in SnS are aligned with the orthorhombic phase of SnS, and the findings are excellently correlated with standard data (JCPDS 390354). The lack of any impurity peaks in the pattern confirms the samples' purity. SnS/rGO has an XRD pattern that is somewhat similar to bare SnS, but with a lower peak amplitude. This may be attributed to the difference in sample sizes. In addition that the calculated lattice parameter values $(a=4.3167 \AA, b=$ $11.0091 \AA, c=4.0021 \AA$ ) of SRG-15 (composite) are also decreased than bare SnS sample $(a=4.1981$ $\AA, b=10.9921 \AA, c=4.0001 \AA$ ). The calculate grain size was $47,41,33$ and $27 \mathrm{~nm}$ for SnS, SRG-5, SRG10 and SRG-15, which is calculated by Debye-Scherer's equation [17].

\subsection{Morphological analysis}

FESEM was used to investigate the morphology of the SnS and their composites, and the related images are shown in Fig.2. The bare GO has a sheet-like morphology with wrinkled base, as seen in Fig. 2a). For the SnS application, the morphology was well crystalline with individual spherical shaped morphology (Fig. 2 b\&c). The SnS nanoparticles have an average diameter of 30-40 nm. These particles are evenly distributed over the surface of the GO sheet (Fig. 2d). The EDS graph given by FESEM confirms the existence of Sn, S, and C elements (Fig. 2e). As a result, the Raman spectra confirmed the formation of the $\mathrm{SnS} / \mathrm{rGo}$ composite.

\subsection{Raman spectra analysis}

The fundamental vibration of a carbon-based metal composite sample was determined using Raman scattering measurements. The Raman spectra of bare $\mathrm{GO}, \mathrm{SnS}$, and their composite samples are seen in Figure 3. Two familiar Raman modes were found in bare GO at 1354 and $1548 \mathrm{~cm}-1$, which correspond to the $D$ and $G$ bands of carbon atoms, respectively [18]. At 110, 170, 185, and $264 \mathrm{~cm}-1$, there are four Raman modes in bare SnS. The Ag mode of SnS is similar to the 110 and $264 \mathrm{~cm}^{-1}$ Raman modes, whereas the other Raman modes are linked to the orthorhombic phase SnS single crystal [19].

\subsection{Optical analysis}

The optical details and band gap energy of the samples is calculated using UV-DRS analysis. The optical absorption spectra of the samples are seen in Fig.4a). It can be shown that the visible light field has a wide spectrum of absorption. The optical absorption edges of rGO and SnS samples were estimated to be 450 and $480 \mathrm{~nm}$, respectively. This absorption was moved to the higher wavelength side, indicating that the band gap energy of the samples was decreasing. The band gap energy of the samples was calculated using the Kubelka - Munk (K-M) relation [20, 21]. The optical band gap energies for rGO, SnS, SRG-5, SRG-10, and SRG-15 were determined to be $2.75,2.53,2.33,2.1$, and $1.85 \mathrm{eV}$, respectively. The PL spectra of the samples obtained with photon excitations at $350 \mathrm{~nm}$ are shown in Figure 5. A large emission peak was clearly noticeable in the visible area at around $450-480 \mathrm{~nm}$, with the strength of emission peaks steadily decreasing for $\mathrm{rGO} / \mathrm{SnS}$ composite samples. This may be because 
recombination photo induced electron-hole pairs are prevented. This is advantageous for improving photo conversion efficiency when exposed to solar rays.

\section{5. $N_{2}$ adsorption-desorption analysis}

The Brunauer-Emmett- Teller (BET) real surface area of the samples was measured using the $\mathrm{N}_{2}$ isothermal adsorption technique (SnS and SRG-15). Figure 6a depicts the graph. The spectra clearly show a type IV isotherm with samples that are mesoporous [22]. As a result, the SRG-15's BET surface area $\left(108.21 \mathrm{~m}^{2} / \mathrm{g}\right)$ is greater than that of bare SnS $\left(84.27 \mathrm{~m}^{2} / \mathrm{g}\right)$. SnS and SRG-15 have equivalent pore sizes of 45.6 and $32.2 \mathrm{~nm}$, respectively (Fig. $6 \mathrm{~b}$ ). The creation of pores between the SnS nanoparticles and GNS, as well as the packing of GNS scattered between the SnS nanoparticles, could both lead to the increased surface region.

\subsection{XPS analysis}

XPS was used to examine the samples' composition and electronic configuration. Figure 7a shows the survey spectrum of SnS/rGO composite (SRG-15) samples. The key elements of Sn, S, and C were clearly evident in the spectra, confirming the formation of the SnS/rGO composite. Two binding energies located at 495.8 and $486.9 \mathrm{eV}$, which belong to the $\mathrm{Sn} 3 \mathrm{~d}$ core-level XPS spectrum, can be specifically $\mathrm{Sn} 3 \mathrm{~d}_{3 / 2}$ and $S n 3 d_{5 / 2}$ peaks, respectively, according to the high resolution spectra of $S n 3 d$ [23]. This means that the $\mathrm{Sn}$ element is entirely in the $4+$ state. There are three binding energies assigned to $\mathrm{O}-\mathrm{C}=\mathrm{O}, \mathrm{C}=\mathrm{O}$, and $\mathrm{C}$ $\mathrm{C} / \mathrm{C}=\mathrm{C}$ groups, respectively, at $289.6,286.1$, and $284.8 \mathrm{eV}$ in C $1 \mathrm{~s}$ [24]. The S $2 \mathrm{p}$ peak is attributed to two peaks at 162.1 and $163.5 \mathrm{eV}$, which are assigned to $S 2 p_{3 / 2}$ and $S 2 p_{1 / 2}$, respectively [25].

\subsection{Photovoltaic studies}

The schematic of a constructed solar cell system as shown in Figure 8a). Under stand illumination, Figure $8 b)$ shows the photocurrent density-voltage $(J-V)$ curves of DSSCs dependent on different CEs (100 $\mathrm{mWcm}^{-2}$ ). Table 1 shows the characteristics of photovoltaic parameters such as short-circuit current density (Jsc), open-circuit voltage (Voc), fill factor FF, and power transfer performance (PCE). Surprisingly, the SnS/rGO (SRG-15) photo anode had a good conversion efficiency (PCE) of $7.72 \%$, which was better than that of SnS nanoparticles used previously (5.21\%). The addition of rGO decreases the resistance and photosensitivity of bare SnS, resulting in improved efficiency. In terms of practical applications, reliability is the most important factor when using the photoanode in photovoltaic systems. As a result, all of the photoelectrodes were subjected to a stability inspection, which is illustrated in Fig. 8c). Just a minor difference in the PCE was noticed after 600 hours of light illumination. The PCE stability of rGO, SnS, SRG-5, SRG-10, and SRG-15 samples was found to be $78,80,82,85$, and $91 \%$, respectively. Figure $8 \mathrm{~d}$ shows a schematic image of the photovoltaic process. The composite sample outperformed bare SnS in terms of photovoltaic efficiency. This may be attributed to the visible light's high emission and high optical clarity. This allows more sunshine to reach the dye molecules and improves DSSC light harvesting. The method of transporting photogenerated electrons from SnS in the presence of graphene 
would be accelerated due to the strong absorption of solar radiation. Additionally, the narrower band distance and lower PL strength were key factors in improving the photovoltaic efficiency of the SnS/rGO composite. As a result, the energetic driving force for electron transfer from graphene to SnS can be decreased while the photosensitive property is increased.

\section{Conclusions}

Chemical reduction was used to successfully treat SnS nanoparticles and SnS/rGO nanocomposite. The orthorhombic step of both bare SnS and SnS/rGO composite samples is shown by all prominent diffraction peaks in the XRD spectra. In addition, the existence of SnS and graphene vibrational modes in the Raman spectrum indicated the development of nanocomposite. UV and PL experiments verified the samples' high optical clarity, band gap energy, and electron-hole pair recombination operation. Doctor blade method has been used to prepare the photoelectrodes and studied the J-V characteristics. The DSSC equipped with SnS/rGO nanocomposite CE achieved a power conversion efficiency (PCE) of $7.72 \%$, which was higher than those of use SnS nanoparticles (5.21\%). Hence, the prepared novel CE can be prepared for high performance photovoltaic device applications.

\section{References}

[1] T. Tsoutsos, N. Frantzeskaki, V. Gekas, Environmental impacts from the solar energy technologies. Energy Policy. 33 (2005) 289-296.

[2] M.Y. Song, K.N. Chaudhari, J. Park, J. Kim, Efficient Pt counter electrode prepared by homogeneous deposition method for dye-sensitized solar cell. Appllied Energy. 100 (2012) $132-137$.

[3] B. Regan, M. Gratzel, A low-cost high-efficiency solar cell based on dye-sensitized colloidal $\mathrm{TiO}_{2}$ films. Nature (Lond.) 353 (1991) 737-740.

[4] M. Gratzel, Solar energy conversion by dye-sensitized photovoltaic cells. Inorganic Chemistry. 44 (2005) 6841-6851.

[5] A. Hagfeldt, G. Boschloo, L. Sun, H.P. Kloo, High performance visible light photocatalytic activity of SnS/rGO composites, Physical Review. 110 (2010) 6595-6663.

[6] Y. Jeon, H. Kim, K. Jim, X. Choi, X. Chen, Hydrothermal induced SnS/carbon nanocomposites and thir structural and optical properties, Nano Energy. 13 (2018) 336-345. 
[7] Lellala Kashinath, K. Namratha, K. Byrappa, Sol-gel assisted hydrothermal synthesis and characterization of hybrid ZnS-RGO nanocomposite for efficient photodegradation of dyes, [6] Alloy. Compd. 695 (2017) 799-809.

[8] S. Yun, Review of photovoltaic materials, Advanced Materials. 26 (2014) 6210-6237.

[9] F. Hao, P. Dong, Q. Luo, L. Li, Design and fabrication of high performance photocatalytic activity of SnS/rGO composites. Energy Environment. 6 (2013) 2003-2019.

[10] P. Poudel, Q. Qiao, Nanodimensional SnS/rGO hybrid composites for dye sensitized solar cell applications, Nano Energy. 4 (2014) 157-175.

[11] K. Novoselov, A.K. Geim, S. Morozov, Two-Dimensional Gas of Mass less Dirac Fermions in Graphene. Nature. 438 (2005) 197-200.

[12] H. Choi, H. Kim, S. Wang, Y. Han, Graphene counter electrodes for dye-sensitized solar cells prepared by electrophoretic deposition, Journal of Materials Chemistry. 21 (2011) $7548-7552$.

[13] R. Jishi, H. Alyahyaei, A facile hydrothermal induced solar cell applications of SnS/rGO hybrid composites, Physical Review B. 78 (2008) 144516.

[14] H. Wang, K. Kalantar-Zadeh, Hybrid nanomaterials of SnS/carbon nanostructures for supercapacitor applications, Nanotechnology. 7 (2012) 699-712.

[15] X. Huang, C. Tan, Z. Yin, Hydrothermal stimulated SnS/rGO composites and its structural, optical and photocatalytic properties, Advanced Materials. 26 (2014) 2185-2204.

[16] Z. Zhang, C. Zhou, M. Jia, Y. Fu, A solvothermal approach for fabricate the SnS/rGO photoelectrodes for solar cell applications, Electrochimica Acta 143 (2014) $305-311$

[17] M. Parthibavarman, V. Hariharan, C. Sekar, V.N. Singh, Effect of Cu on structural, optical and electrochemical properties of $\mathrm{SnO}_{2}$ nanoparticles, Journal of optoelectronics and 
advanced materials 12 (2010) 1894-1898.

[18] J. Wu, B. Liu, M. Ren, W. Gong, L. Qin, A one-pot hydrothermal induced SnS/rGO composite materials and its structural, optical and photocatalytic properties, Applied Surface Science. 50 (2014) 456-461.

[19] Arpita Paul Chowdhury., Baban H. Shambharkar., Sachin G. Ghugal, S. Suresh, Hydrothermal stimulated SnS/rGO composites and its structural, optical and photocatalytic properties, RSC Advances. 6 (2016) 18290-108297

[20] H.S. Jadhav, S.M. Pawar, A.H. Jadhav, G.M. Thora, J.G. Seo, Hierarchical Mesoporous 3D Flower-like SnS/NF for High-Performance Electrochemical Energy Storage, Journal of Power Sources. 6 (2016) 1120-1127.

[21] Ali A. Ensafi, S.E. Moosavifard, B. Rezaeia, S.K. Kaverlavania, Engineering onion-like nanoporous SnS hollow spheres derived from bimetal-organic frameworks for highperformance asymmetric supercapacitors. Journal of Materials Science. 56 (2018) 1049710506

[22] K. Qiu, M. Lu, Y. Luo, X. Du, Engineering hierarchical nanotrees with SnS trunks and $\mathrm{NiO}$ branches for high-performance supercapacitors. Journal of Materials Science. 5 (2017) 5820-5828.

[23] S. Vijayakumar, S.H. Lee, K.S. Ryu, Hierarchical SnS nanobelts as a supercapacitor electrode with high areal and specific capacitance. Electrochim Acta. 182 (2015) 979-986 [24] M. Karthik, M. Parthibavarman, A. Kumaresan, S. Prabhakaran, One-step microwave synthesis of pure and $\mathrm{Mn}$ doped $\mathrm{WO}_{3}$ nanoparticles and its structural, optical and electrochemical properties. Journal of Materials Science: Materials in Electronics. 28 (2017) 6635-6642

[25] R. Jose, S.G. Krishnan, B. Vidyadharan, I.I. Misnon, M. Harilal, R.A. Aziz, J. Ismail 
M.M. Yusoff, Supercapacitor electrodes delivering high energy and power densities.

Materials Today: Proceedings. 3 (2016) S48 - S56.

Figures

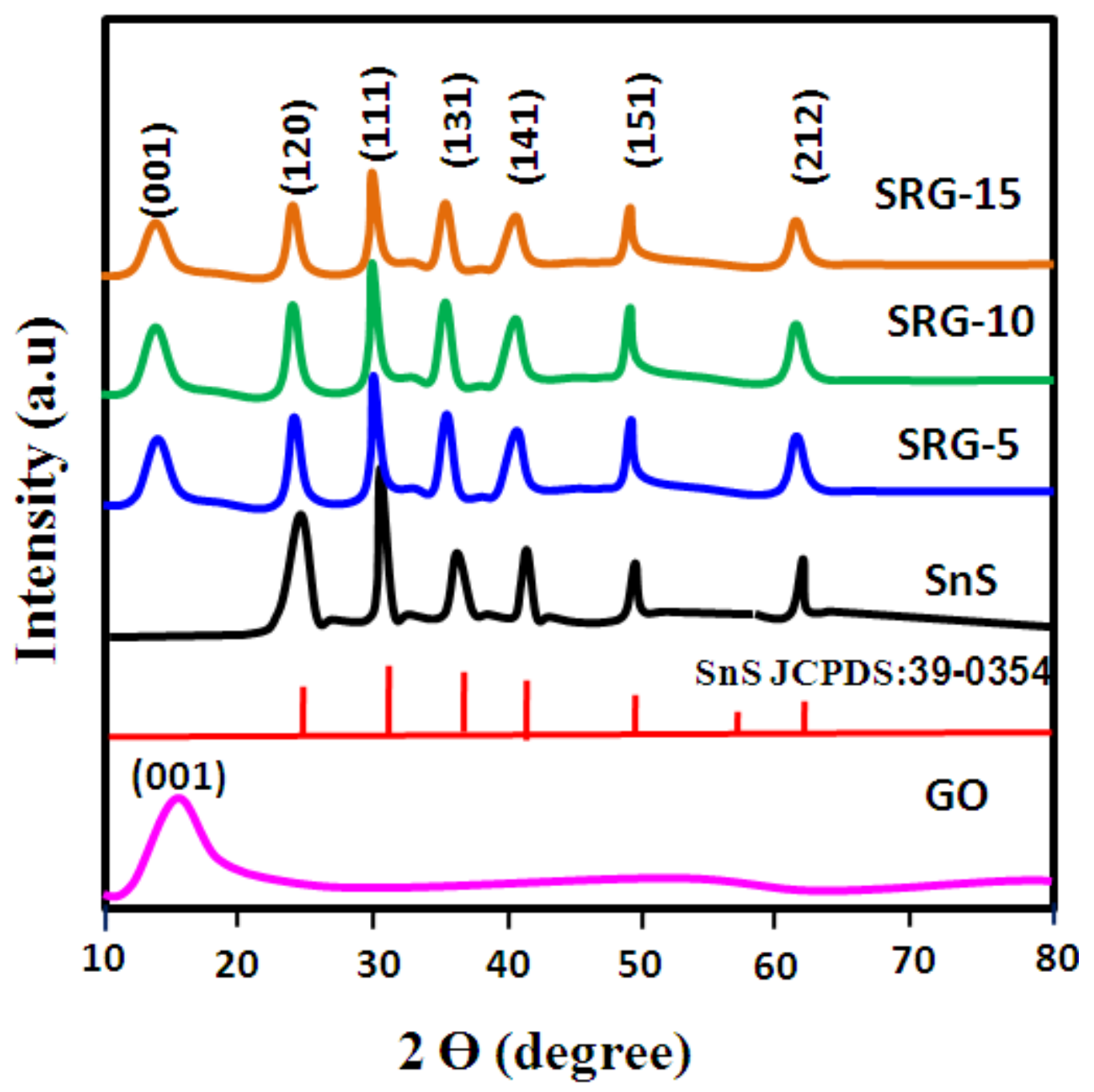

Figure 1

Powder X-ray diffraction pattern of pure rGO, SnS and SnS/rGO composite samples 


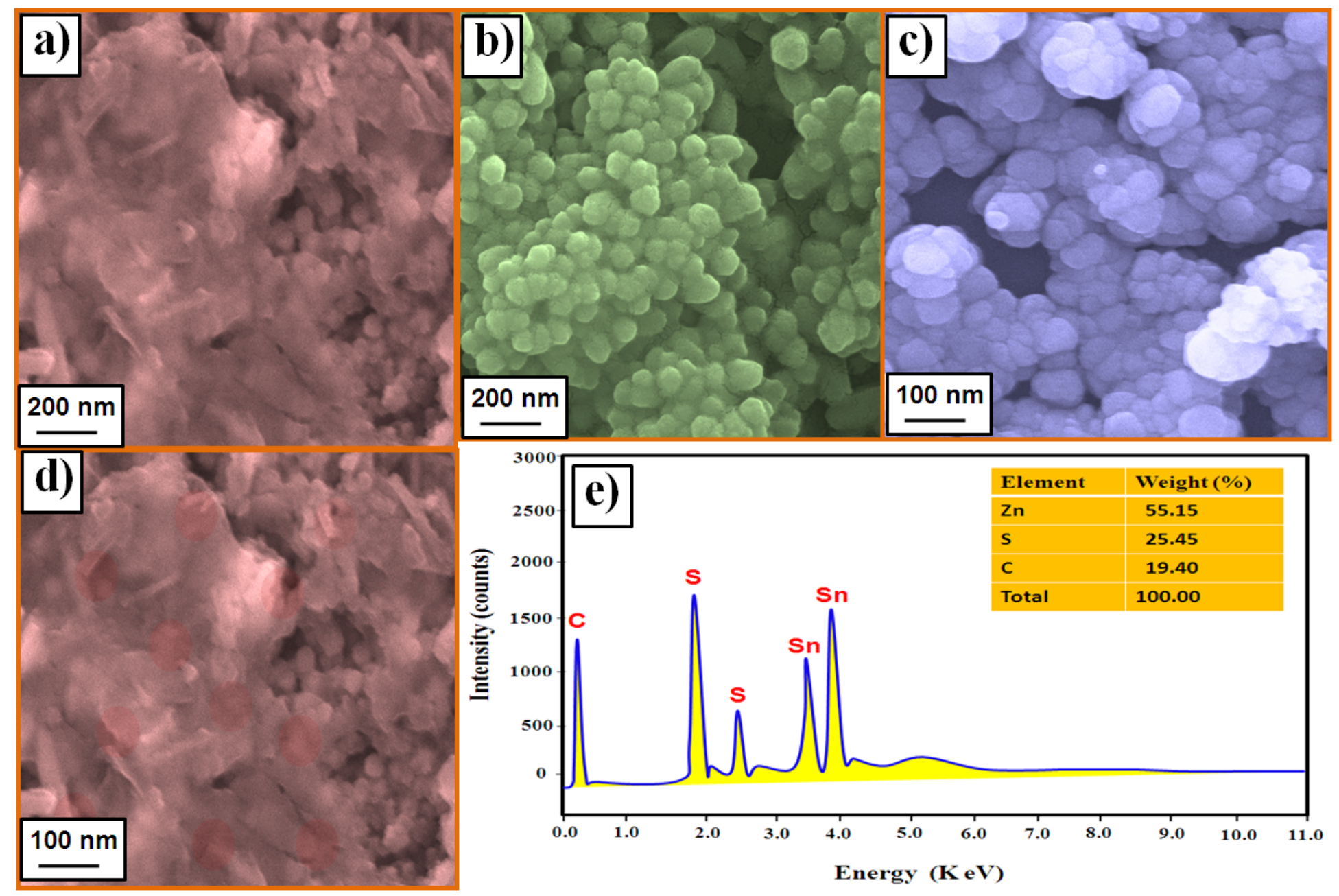

Figure 2

FESEM images of a) rGO b) and c) different magnification images of SnS; d) FESEM image of SnS/rGO (SRG-15); e) and f) EDS images of pure SnS and SRG-15 samples respectively. 


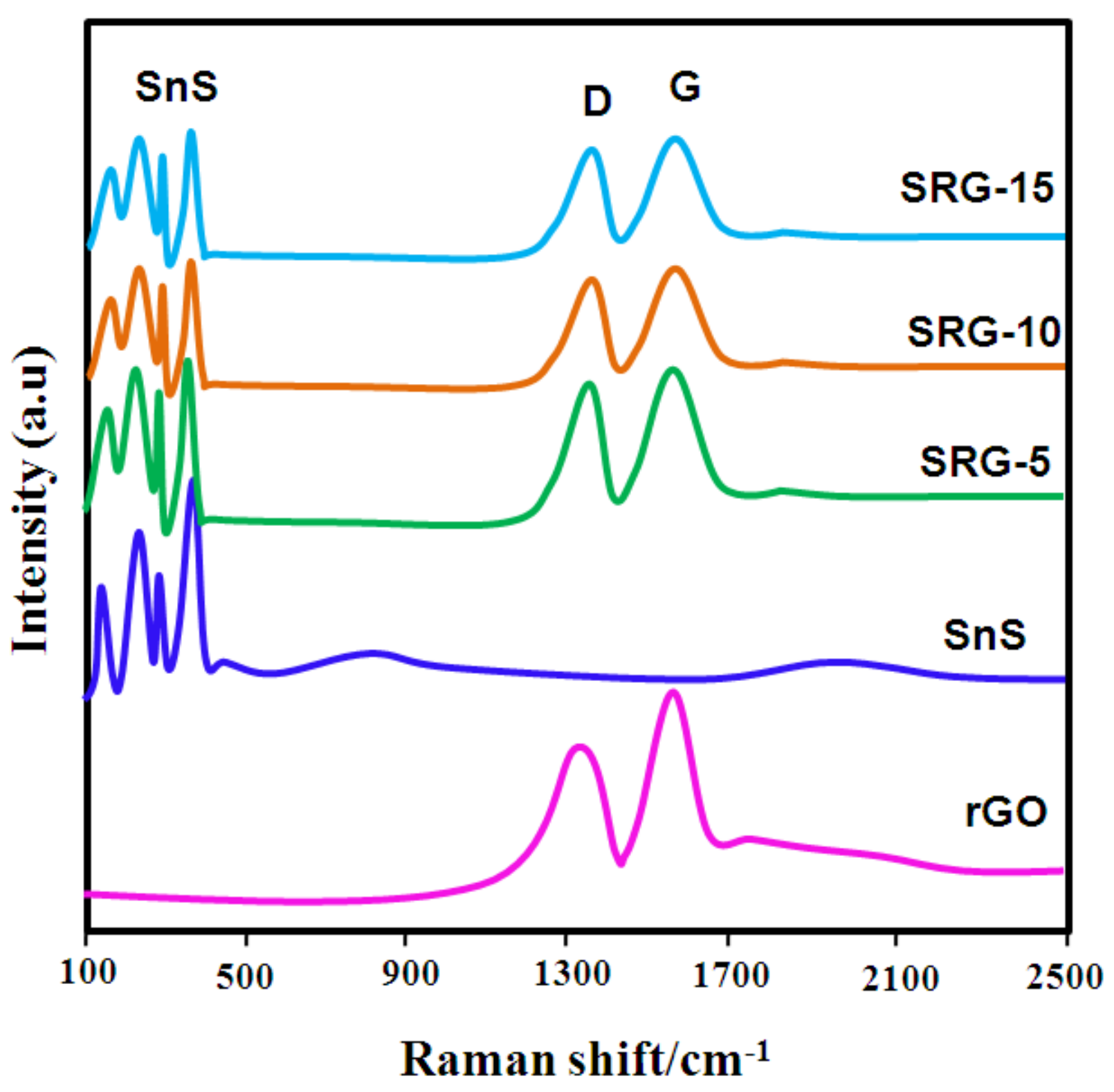

Figure 3

Raman spectra of pure rGO, SnS and various SnS/rGO composite samples 


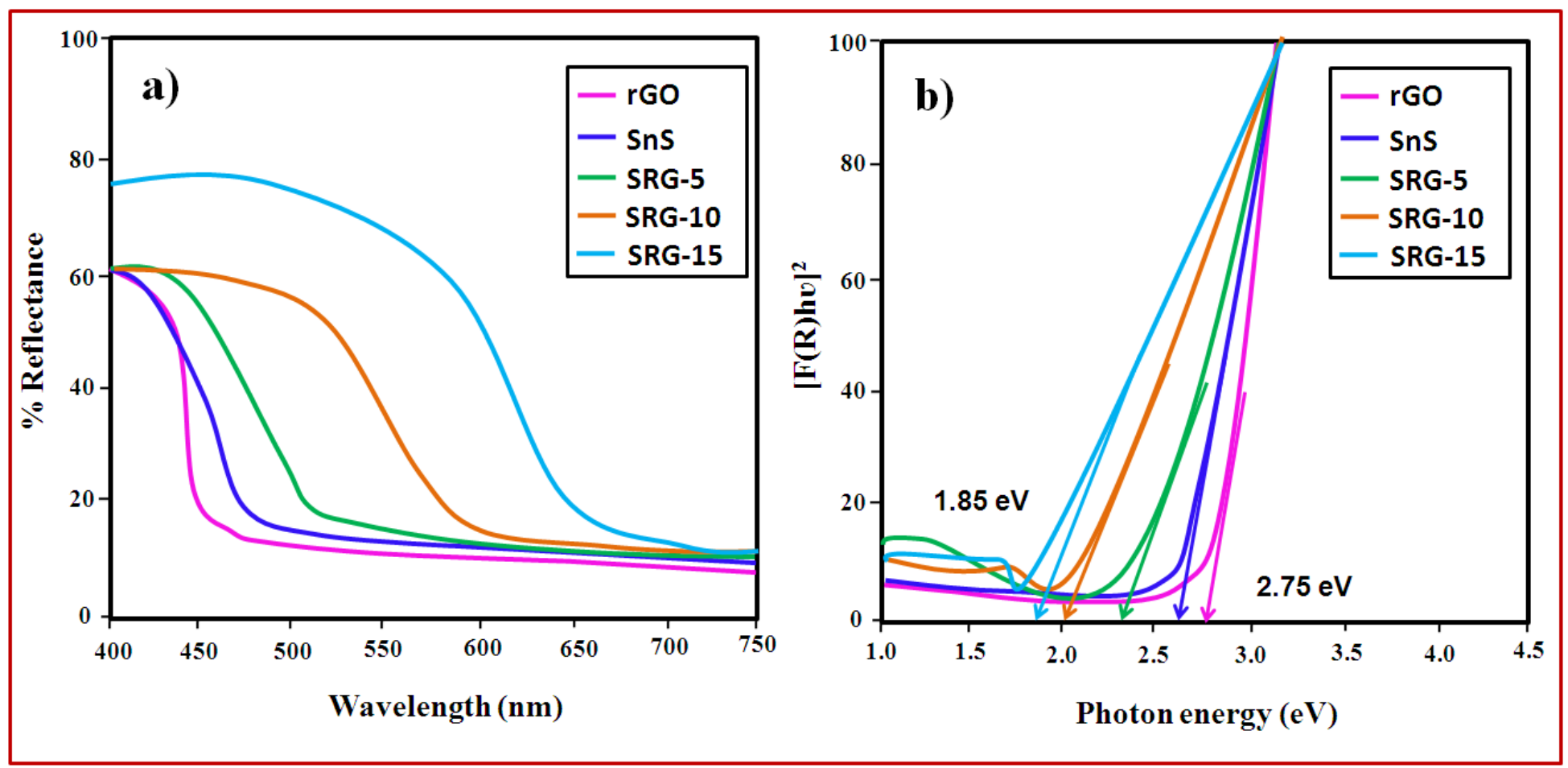

Figure 4

UV-Vis DRS spectra of pure SnS and SnS/rGO composite samples a) Reflectance spectra b) KubelkaMunk model 


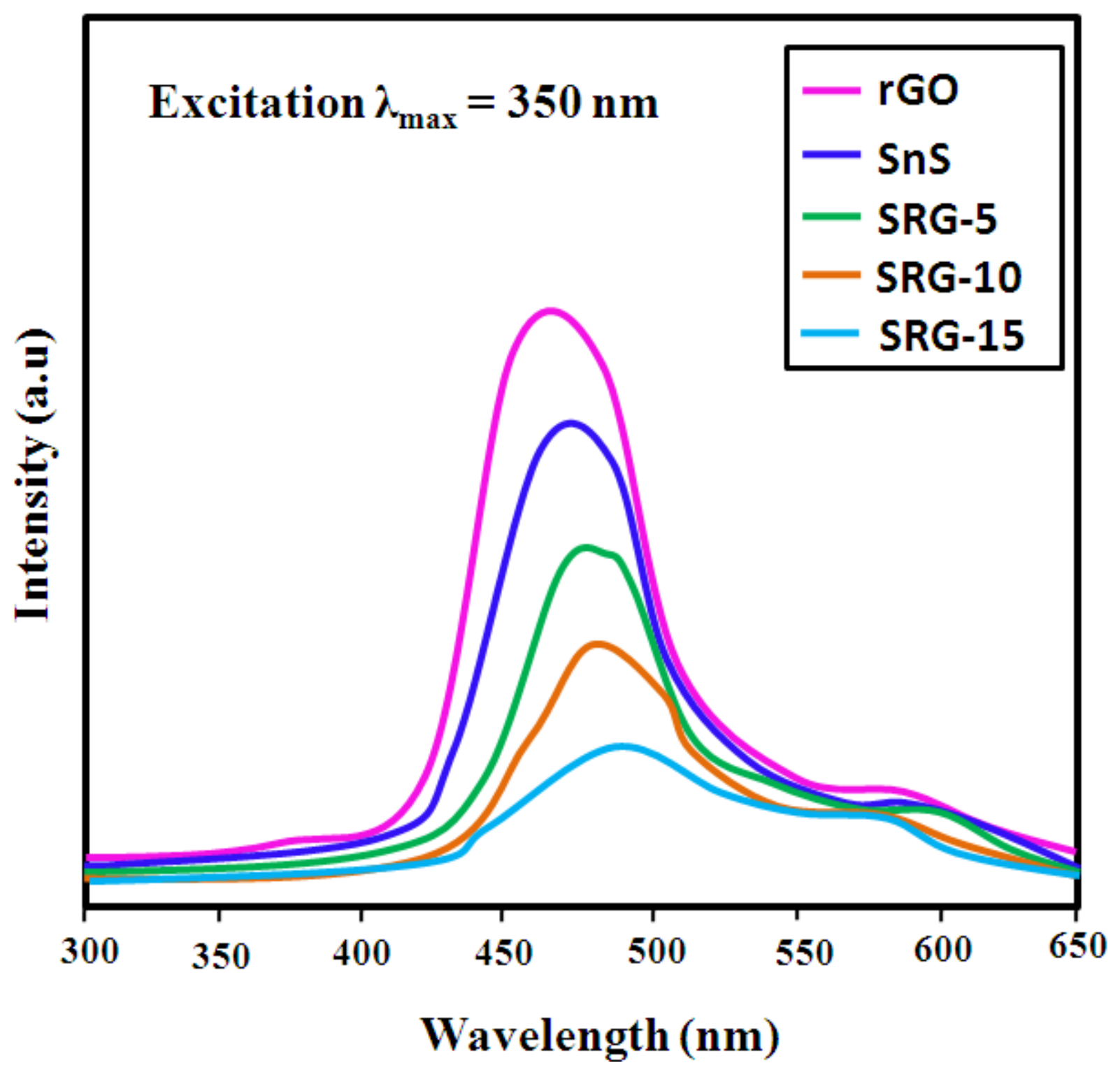

Figure 5

Photoluminescence spectra of pure rGO, SnS and SnS/rGO composite samples 


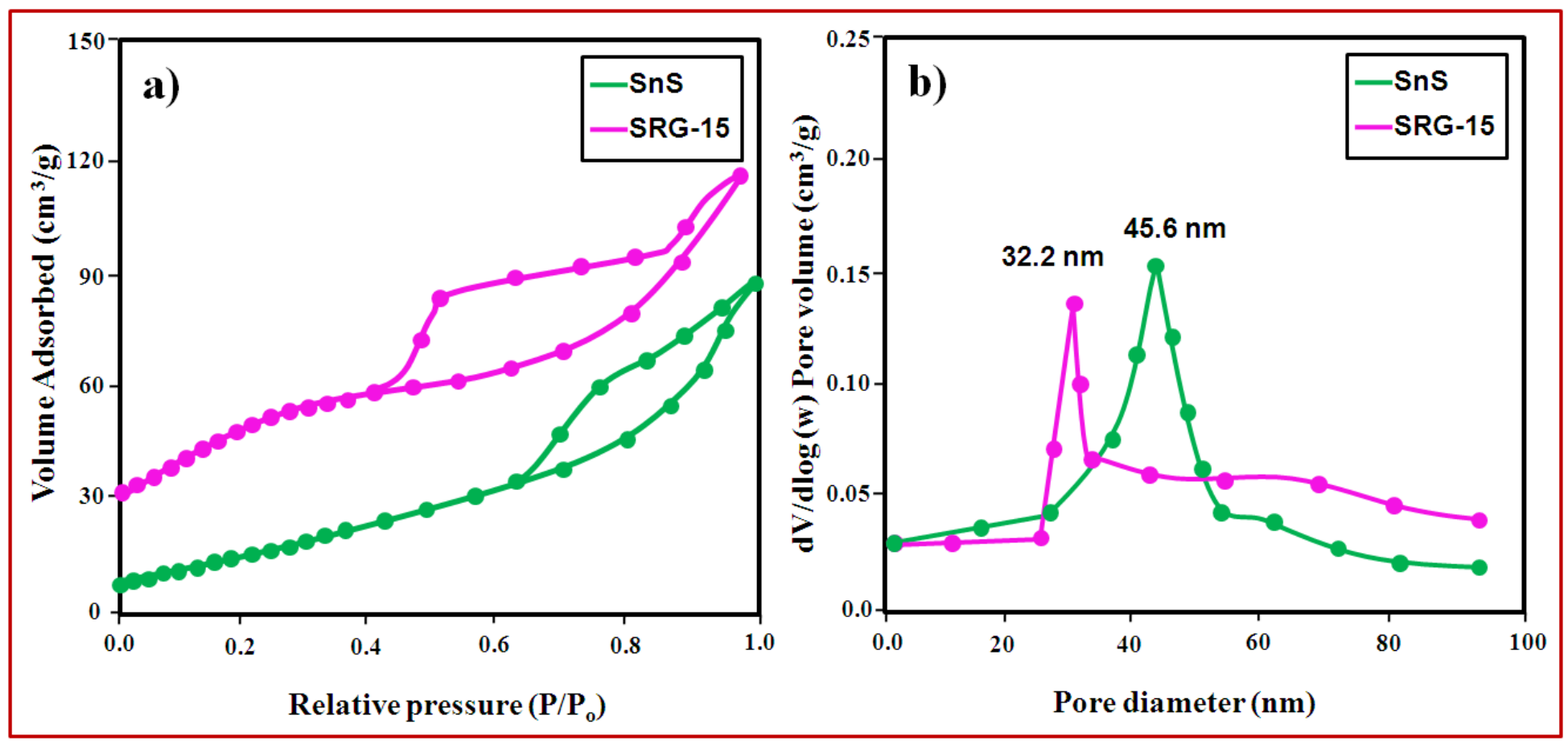

Figure 6

a) N2 adsorption and desorption analysis pure SnS and SRG-15 composite samples b) corresponding pore size distribution 


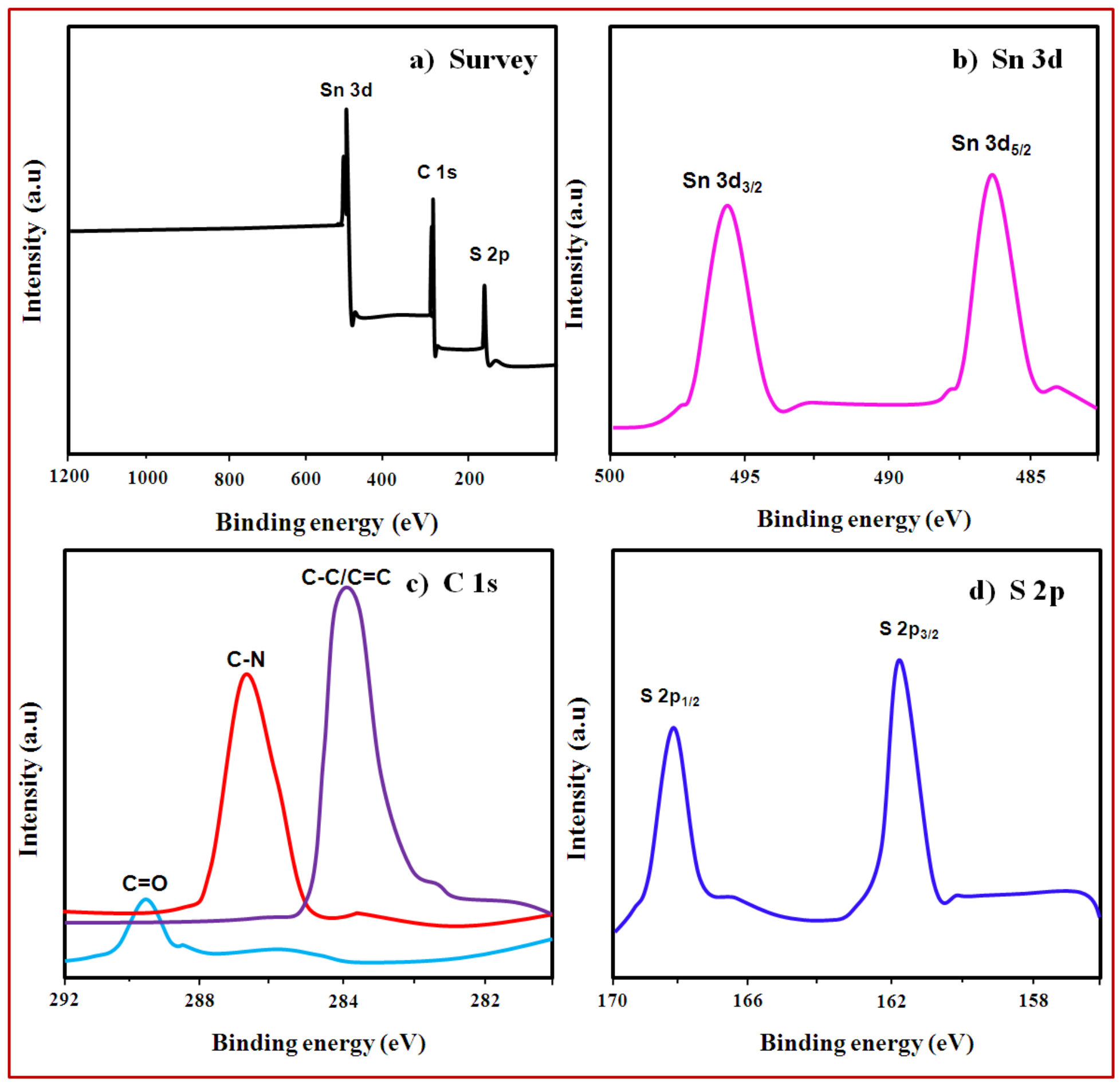

Figure 7

XPS spectra of SnS/rGO a) survey; b) C $1 s$; c) Sn 2p; and d) S 2p 


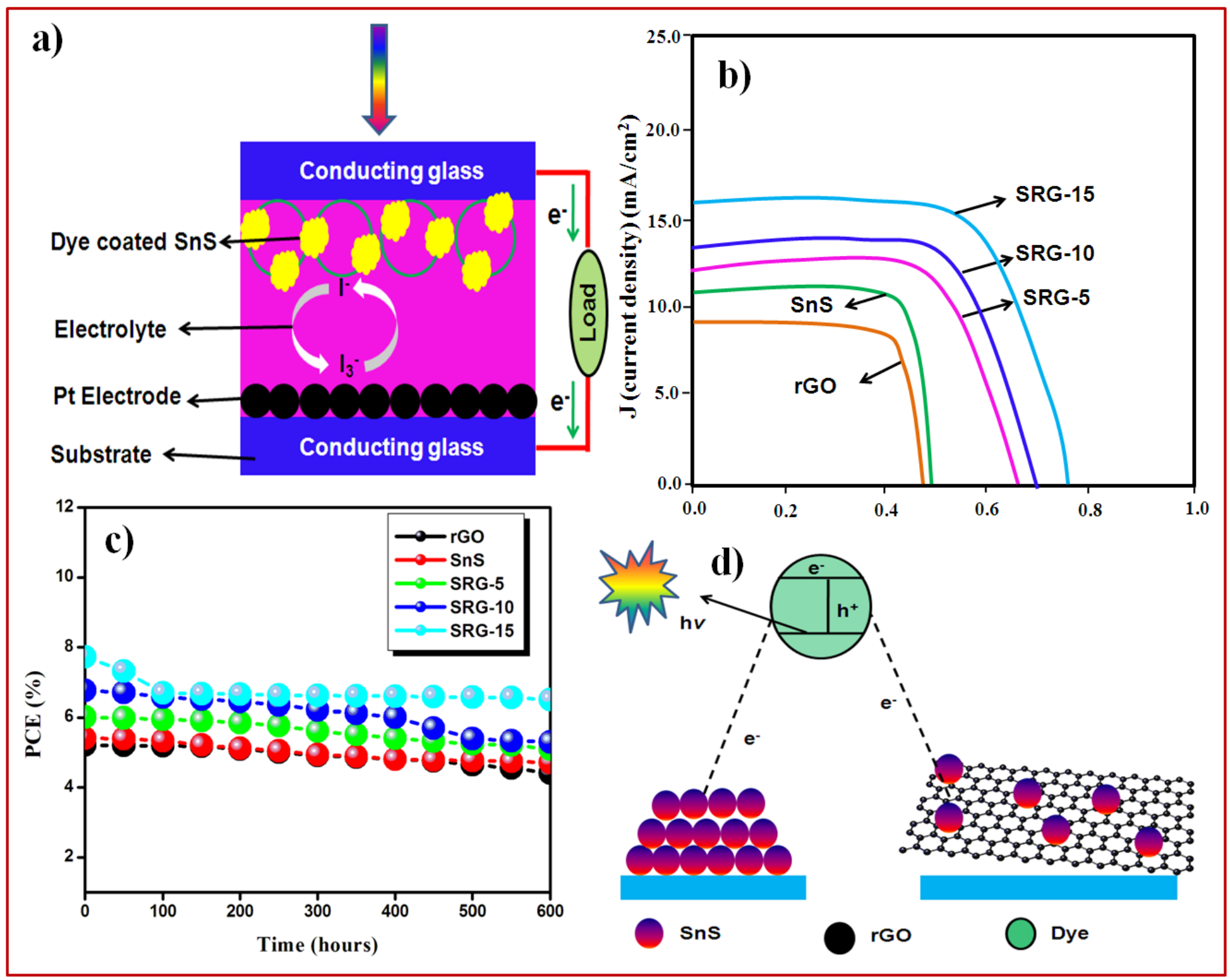

Figure 8

a) Schematic representation of the device; b) Photovoltaic performance of un-doped SnS and SnS/rGO composite samples; c) Stability test and d) Schematic representation for photovoltaic conversion mechanism of $\mathrm{SnS} / \mathrm{rGO}$ 